\title{
Efectos del entrenamiento de fuerza sobre la condición física en niños y niñas con sobrepeso y obesidad: una revisión sistemática Effects of strength training on the fitness in boys and girls with overweight and obesity: a systematic review \\ *Luis Le-Cerf Paredes, **Pablo Valdés-Badilla, *Eduardo Guzmán Muñoz \\ *Universidad Santo Tomás (Chile), **Universidad Católica del Maule (Chile)
}

Resumen. Introducción: Debido a las preocupantes cifras de obesidad infantil y los riesgos para la salud que significa tener este estado nutricional no saludable, nuestro objetivo fue realizar una revisión sistemática para determinar los efectos del entrenamiento de fuerza muscular sobre la condición física general de niños y niñas con sobreopeso y obesidad. Métodos: Se utilizaron las bases de datos PubMed, Science direct, Scopus, SciELO y Google Scholar. Los estudios que cumplieron los criterios de inclusión fueron valorados en cuanto a su calidad metodológica a través de la escala PEDro. Resultados: Los estudios señalan que el entrenamiento de fuerza cuando es aplicado de manera aislada aumenta la fuerza muscular, flexibilidad, potencia muscular, agilidad y capacidad cardiorrespiratoria en niños y niñas con sobrepeso y obesidad. Cuando el entrenamiento de fuerza se combinó con actividades aeróbicas se observaron aumentos significativos de la fuerza muscular, capacidad cardiorrespiratoria y flexibilidad Conclusiones: A pesar de la diversidad de los estudios, el entrenamiento de fuerza muscular parece tener un efecto positivo en la condición física general de los niños y niñas con sobrepeso y obesidad.

Palabras claves: obesidad infantil, condición física, entrenamiento de fuerza, entrenamiento de resistencia.

\begin{abstract}
Introduction: Due to the worrying figures for childhood obesity and the health risks of having this unhealthy nutritional state, our objective was to carry out a systematic review to determine the effects of muscle strength training on the general physical condition of boys and girls overweight and obese. Methods: PubMed, Science direct, Scopus, SciELO and Google Scholar databases were used. The studies that met the inclusion criteria were assessed for their methodological quality using the PEDro scale. Results: Studies indicate that strength training when applied in isolation increases muscle strength, flexibility, muscle power, agility, and cardiorespiratory capacity in overweight and obese boys and girls. When strength training was combined with aerobic activities, significant increases in muscle strength, cardiorespiratory capacity and flexibility were observed. Conclusions: Despite the diversity of studies, muscle strength training seems to have a positive effect on the general physical condition of overweight and obese boys and girls.
\end{abstract}

Key words: childhood obesity, physical fitness, strength training, resistance training.

\section{Introducción}

El sobrepeso y la obesidad se definen como una acumulación anormal excesiva de grasa que puede ser perjudicial para la salud (Ng et al., 2014). En el año 2010, la prevalencia de sobrepeso y obesidad entre los niños en edad preescolar aumentó en un 60\% desde 1990, afectando a unos 43 millones de niños en todo el mundo (de Onis, Blossner, \& Borghi, 2010). En Estados Unidos de América, el 29\% de los niños y adolescentes presentan exceso de peso (Ng et al., 2014), mientras que en España la prevalencia alcanza a un $46 \%$ con mayor presencia en varones (Sanchez-Cruz, Jimenez-Moleon, FernandezQuesada, \& Sanchez, 2013). Por otra parte, en algunos

Fecha recepción: 16-02-21. Fecha de aceptación: 24-06-21

Eduardo Guzman Muñoz

eguzmanm@santotomas.cl países de América Latina las tasas son cercanas al 50\% de sobrepeso y obesidad en niños situados entre los 5 y 9 años (Ramirez-Izcoa et al., 2017; Tobarra, Castro, \& Badilla, 2015).

La causa fundamental del sobrepeso y la obesidad corresponde a un desequilibrio energético entre calorías consumidas y gastadas, relacionándose con un mayor riesgo de padecer enfermedades metabólicas y cardiovasculares (Bastien, Poirier, Lemieux, \& Despres, 2014; Rajmil et al., 2016). El exceso de peso en la infancia se ha asociado con un déficit en el desarrollo de las habilidades motoras y condición física, lo que redunda en menor participación en actividades físicas y deportivas (Guzmán-Muñoz, Valdes-Badilla, MendezRebolledo, Concha-Cisternas, \& Castillo-Retamal, 2019; Guzmán-Muñoz \& Castillo-Retamal, 2020; GuzmánMuñoz, Valdés-Badilla, Concha-Cisternas, MéndezRebolledo, \& Castillo Retamal, 2020; Kakebeeke et 
al., 2017). Además, el aumento de peso genera alteraciones posturales y musculosqueléticas que pueden tener un importante impacto en la calidad de vida de los niños (Martins, Cardoso, Honorio \& Silva, 2020).

Específicamente, la fuerza muscular ha sido considerada fundamental para el desarrollo motor y representa un aspecto importante en el estado de salud (He et al., 2019; Wind, Takken, Helders, \& Engelbert, 2010). Una disminución de la fuerza muscular en niños puede repercutir en limitaciones funcionales (Takken et al., 2003) y, además, incrementar el riesgo de enfermedades cardiometabólicas (Fraser et al., 2017; Grontved et al., 2015). Estudios señalan que el entrenamiento de la fuerza es beneficioso para niños y adolescentes con sobrepeso y obesidad, ya que disminuye el tejido adiposo a nivel central, genera cambios favorables en el perfil lipídico, mejora la función cardiovascular y disminuye los factores de riesgos metabólicos (Fraser et al., 2017; Grontved et al., 2015; Timpka, Petersson, Zhou, \& Englund, 2014). En este sentido, el entrenamiento de fuerza favorece cambios musculares que aumentan el gasto calórico contribuyendo con la disminución de peso corporal. Estos cambios son intracelulares y promueven la síntesis de proteínas estructurales y funcionales responsables de mejorar la capacidad funcional del músculo, lo cual, podría contribuir en la eficiencia del consumo máximo de oxígeno (Prieto-González, Sagat, BenBrahim \& Sedlacek, 2020). Por otra parte, el entrenamiento de fuerza como método de acondicionamiento físico favorece la adherencia en niños y niñas con sobrepeso y obesidad, ya que brinda la oportunidad para que todos los niños, independientemente de su tamaño corporal, lo desarrollen con éxito y se sientan satisfechos con su desempeño (Faigenbaum, Perez, \& Naclerio, 2011; Sun, Wang, \& Wang, 2015). Se ha propuesto que los niños con sobrepeso y obesidad tienden a disfrutar del entrenamiento de fuerza porque generalmente se caracteriza por períodos cortos de actividad física intercalados con breves períodos de pausa entre series (Faigenbaum et al., 2011).

Pese a que el entrenamiento de fuerza muscular ha mostrado ser esencial para mejorar la condición física general, sus resultados se han reportado mayormente en adolescentes y adultos. Por lo tanto, el objetivo de esta investigación fue revisar sistemáticamente los estudios que han empleado el entrenamiento de fuerza como parte de una intervención para niños y niñas con sobrepeso y obesidad, examinando el efecto sobre la condición física general (p.e. fuerza, capacidad cardiorrespiratoria, flexibilidad, entre otras).

\section{Material y métodos}

\section{Estrategia de búsqueda}

Se realizó una búsqueda comprensiva, exhaustiva y estructurada siguiendo las recomendaciones PRISMAP (Moher et al., 2015) en las siguientes bases de datos: PubMed, Science Direct, Scopus, SciELO Citation Index y Google Scholar, entre el 21 de septiembre y 23 de octubre de 2020. Todos los artículos fueron descargados y se realizó una referencia cruzada manual para identificar los duplicados. Los títulos y resúmenes se seleccionaron para una revisión posterior del texto completo. Los artículos incluidos en esta búsqueda fueron desde el año 2010 al 2020. Se utilizaron las siguientes palabras claves para la búsqueda de información en las bases de datos ya mencionadas: «resistance training», «strength training», «physical fitness», "physical conditioning», «obesity children», «obesity pediatric» $\mathrm{y}$ «child obesity». Se combinaron de diversas formas con los términos booleanos «OR»y «AND».

\section{Selección de estudios}

Se consideraron estudios experimentales, es decir, ensayos controlados aleatorizados (ensayos clínicos), no aleatorizados (cuasiexperimentales) y pre-experimentales. Los artículos fueron incluidos en la revisión sistemática si cumplían los siguientes criterios de inclusión: i) artículos en idioma inglés o español; ii) artículos cuya población incluya niños y niñas con sobrepeso y obesidad situados entre los 6 a 12 años de edad; iii) artículos que contengan intervención de entrenamiento de fuerza en niños con sobrepeso y obesidad (el entrenamiento de fuerza pudo ser la única intervención o ser aplicado combinadamente con otras intervenciones como, por ejemplo, ejercicio aeróbico); iv) artículos que tuviesen el reporte de resultados en al menos una variable de la condición física (p.e. fuerza muscular, capacidad cardiorrespiratoria, flexibilidad, entre otras).

Los criterios de exclusión fueron los siguientes: i) estudios que no correspondían a publicaciones originales de investigación (p.e. cartas al editor, traducciones, notas, revisiones de libros); ii) artículos duplicados; iii) artículos de revisión (p.e. meta-análisis, revisiones sistemáticas, revisiones narrativas); y iv) estudios de caso (es decir, estudios que usan sólo una persona).

La búsqueda fue realizada por dos investigadores de forma independiente, en cada una de las bases de datos anteriormente mencionadas y con la estrategia de búsqueda presentada. Los artículos fueron seleccionados si tras leer el título y/o resumen podrían cumplir los cri- 
terios de elegibilidad. Se obtuvo el texto completo de los artículos seleccionados, se aplicaron rigurosamente los criterios de inclusión y exclusión, y, por último, se decidió si eran pertinentes para la evaluación final.

\section{Evaluación y calidad de los estudios}

Los estudios seleccionados fueron evaluados a través de la escala PEDro. Esta escala evalúa la calidad metodológica de las investigaciones, considerando 11 puntos que incluye procedimiento de cegamiento, el análisis estadístico, información sobre la aleatorización y la presentación de los resultados en la investigación evaluada (Ribeiro, Gómez-Conesa, \& Montesinos, 2010). El criterio 1 evalúa la validez externa y no está incluido en el resultado final. Del criterio 2 al 11 se evalúa la validez interna del artículo con un sistema de puntuación estandarizado (rango de 0 a 10). Estudios con una puntuación e» 5 en la escala PEDro fueron considerados con una calidad metodológica media/alta (Moseley, Herbert, Sherrington, \& Maher, 2002).

\section{Síntesis de datos}

Se obtuvieron y analizaron los siguientes datos de los estudios seleccionados: i) muestra: número de participantes, ii) Edad media de la muestra, iii) Estado nutricional: media del IMC, iv) Protocolo de entrenamiento, v) Duración de entrenamiento (frecuencia y tiempo por sesión), vi) variables de condición física analizadas, y vi) principales resultados obtenidos.

\section{Resultados}

El proceso de búsqueda se detalla en la Figura 1. Se identificaron un total de 1.210 artículos en las bases de datos PubMed, Science Direct, Scopus, SciELO Citation Index y Google Scholar. En la fase de screening se eliminaron los duplicados y los estudios fueron filtrados seleccionando el título, resumen y palabras clave obteniendo como resultado 63 referencias. Un total de 28 estudios fueron analizados a texto completo, siendo 5 excluidos por no estar enfocados en entrenamiento de fuerza, 4 porque no especifican el tipo de entrenamiento aplicado, 9 porque no evaluaron variables relacionadas con la condición física y 2 porque no especificaron el estado nutricional de los niños intervenidos. Ocho estudios cumplieron con todos los criterios de selección (Alberga, Farnesi, Lafleche, Legault, \& Komorowski, 2013; Bruno et al., 2018; Calcaterra et al., 2013; Crouter, Salas, \& Wiecha, 2017; Farris, Taylor, Williamson, \&
Robinson, 2011; Horsak et al., 2019; Nobre et al., 2017; Vasquez et al., 2013). De ellos, 4 corresponden a ensayos controlados aleatorizados (Alberga et al., 2013; Bruno et al., 2018; Horsak et al., 2019; Nobre et al., 2017) y 4 son estudios preexperimentales (pre-post sin grupo control) (Calcaterra et al., 2013; Crouter et al., 2017; Farris et al., 2011; Vasquez et al., 2013).

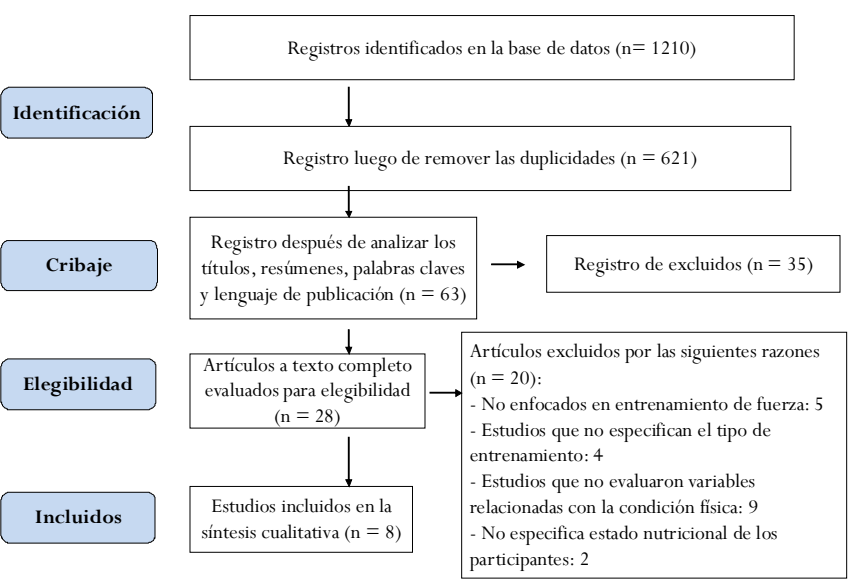

En la Tabla 1 se presentan los resultados de la evaluación de calidad metodológica, donde dos estudios obtuvieron una puntuación 7/10 (Bruno et al., 2018; Horsak et al., 2019), uno 6/10 (Alberga et al., 2013) y uno 5/ 10 (Nobre et al., 2017). El resto de los estudios obtuvo una puntación menor a 5/10 (Calcaterra et al., 2013; Crouter et al., 2017; Farris et al., 2011; Vasquez et al., 2013).

Tabla 1.

Escala de PEDro para la valoración metodológica de los estudios incluidos

\begin{tabular}{|c|c|c|c|c|c|c|c|c|c|c|c|c|}
\hline \multirow[b]{2}{*}{ Estudio } & \multicolumn{11}{|c|}{ Criterios } & \multirow[b]{2}{*}{ Total } \\
\hline & $1 *$ & 2 & 3 & 4 & 5 & 6 & 7 & 8 & 9 & 10 & 11 & \\
\hline Horsak et al., 2019 & 1 & 1 & 0 & 1 & 0 & 0 & 1 & 1 & 1 & 1 & 1 & 7 \\
\hline Bruñó et al., 2018 & 1 & 1 & 1 & 1 & 0 & 0 & 1 & 0 & 1 & 1 & 1 & 7 \\
\hline Nobre et al., 2017 & 1 & 1 & 0 & 1 & 0 & 0 & 0 & 1 & 0 & 1 & 1 & 5 \\
\hline Crouter et al., 2016 & 1 & 0 & 0 & 0 & 0 & 0 & 0 & 1 & 0 & 1 & 1 & 3 \\
\hline Alberga et al., 2013 & 1 & 1 & 0 & 1 & 0 & 0 & 0 & 1 & 1 & 1 & 1 & 6 \\
\hline Calcaterra et al., 2013 & 1 & 0 & 0 & 0 & 0 & 0 & 0 & 1 & 0 & 1 & 1 & 3 \\
\hline Vásquez et al., 2013 & 1 & 0 & 0 & 1 & 0 & 0 & 0 & 1 & 0 & 1 & 1 & 4 \\
\hline Farris et al., 2011 & 1 & 0 & 0 & 0 & 0 & 0 & 0 & 1 & 0 & 1 & 1 & 3 \\
\hline
\end{tabular}

Las características de la muestra, protocolos de entrenamiento, variables de condición física y principales resultados obtenidos por los estudios se resumen en la Tabla 2.

\section{Muestra}

En relación con la muestra, un estudio contaba con una muestra menor a 20 participantes (Alberga et al., 2013), dos con 20 a 50 (Calcaterra et al., 2013; Crouter et al., 2017), cuatro con 50 a 100 (Bruno et al., 2018; Farris et al., 2011; Horsak et al., 2019; Nobre et al., 2017) y uno con una muestra mayor a 100 participantes (Vasquez et al., 2013), lo que totalizó 395 participantes. 


\begin{tabular}{|c|c|c|c|c|c|c|c|}
\hline Autor & Muestra & Edad & $\begin{array}{l}\text { Estado } \\
\text { nutricional }\end{array}$ & Protocolo de entrenamiento & $\begin{array}{l}\text { Duración de } \\
\text { entrenamiento }\end{array}$ & Variables de condición física & Principales resultados \\
\hline $\begin{array}{l}\text { Horsak et } \\
\text { al., } 2019 .\end{array}$ & $\begin{array}{l}51 \text { niños y adolescentes } \\
\text { divididos en } 2 \text { grupos } \\
\text { (grupo experimental }=26 / \\
\text { grupo control } n=25 \text { ). }\end{array}$ & $\begin{array}{l}\text { Entre } 10 \mathrm{y} \\
18 \text { años. }\end{array}$ & Obesidad & $\begin{array}{l}\text { Entrenamiento de fuerza }+ \text { ejercicios } \\
\text { neuromusculares } \\
\text { Se utilizó el peso corporal para el } \\
\text { entrenamiento músculos de rodilla y cadera. } \\
\text { Intensidad: se monitorizo con escala de } \\
\text { esfuerzo percibido de } 0 \text { a } 10 \text {, pero no se } \\
\text { especifica nivel utilizado }\end{array}$ & $\begin{array}{l}12 \text { semanas, } 2 \text { sesiones } \\
\text { por semana, duración } 60 \\
\text { minutos cada sesión. }\end{array}$ & $\begin{array}{l}\text { - Fuerza muscular de cuádriceps y } \\
\text { abductores de cadera }\end{array}$ & $\begin{array}{l}\text { Aumenta fuerza de cuádriceps } \\
\text { en } 20 \% \text { y fuerza de } \\
\text { abductores en } 30 \% \text {. Sin } \\
\text { embargo, cambio fue } \\
\text { significativo solo en } \\
\text { abductores. } \\
\text { En grupo control fuerza } \\
\text { aumento menos de un } 5 \% \\
\end{array}$ \\
\hline $\begin{array}{l}\text { Bruñó et } \\
\text { al., } 2018 .\end{array}$ & $\begin{array}{l}52 \text { niños } \mathrm{y} \text { adolescentes } \\
\text { divididos en } 3 \text { grupos } \\
\text { (Grupo ejercicios impresos } \\
\mathrm{n}=18 / \text { Grupo plataforma } \\
\text { web } \mathrm{n}=18 \text { / Grupo } \\
\text { plataforma web y soporte } \\
\text { adicional } \mathrm{n}=16 \text { ) }\end{array}$ & $\begin{array}{l}\text { Entre } 9 \text { y } 1 \\
\text { años. }\end{array}$ & $\begin{array}{r}\text { 6Sobrepeso } \\
\text { y obesidad }\end{array}$ & $\begin{array}{l}\text { Entrenamiento de fuerza }+ \text { actividad } \\
\text { aeróbica. } \\
\text { Entrenamiento tipo circuito con } 10 \text { ejercicios } \\
\text { de fuerza. Se utilizaron mancuernas y peso } \\
\text { corporal para el entrenamiento } \\
\text { Intensidad: baja intensidad con un alto } \\
\text { número de repeticiones. No se especifica uso } \\
\text { de escala para valorar el esfuerzo percibido. }\end{array}$ & $\begin{array}{l}3 \text { meses, } 60 \text { sesiones, } 5 \\
\text { sesiones semanales, } 60 \\
\text { s minutos de duración cada } \\
\text { sesión. }\end{array}$ & $\begin{array}{l}\text { - Fuerza muscular (dinamometría } \\
\text { prensión manual) } \\
\text { a- Capacidad } \mathrm{CR}\left(\mathrm{VO}_{2} \text { peak) }\right.\end{array}$ & $\begin{array}{l}\text { - Fuerza de prensión manual y } \\
\text { capacidad CR aumentaron en } \\
\text { los tres grupos de } \\
\text { intervención, pero no } \\
\text { significativamente. }\end{array}$ \\
\hline $\begin{array}{l}\text { Nobre et } \\
\text { al., } 2017 .\end{array}$ & $\begin{array}{l}59 \text { niños, divididos en } 2 \\
\text { grupos (grupo } \\
\text { experimental } n=40 / \text { grupo } \\
\text { control } n=19) .\end{array}$ & $\begin{array}{l}\text { Entre } 7 \text { y } 9 \\
\text { años } \\
\text { o }\end{array}$ & $\begin{array}{l}\text { Sobrepeso } \\
\text { y obesidad. }\end{array}$ & $\begin{array}{l}\text { Entrenamiento de fuerza (tipo pliométrico). } \\
\text { Se utilizaron cajones de } 10 \text { a } 40 \mathrm{~cm} \text { para } \\
\text { entrenamiento de extremidad inferior. } \\
\text { Intensidad: } 5 \text { series de } 10-24 \text { repeticiones } \\
\text { progresivas a lo largo de las semanas. Total de } \\
\text { saltos: } 50 \text { en primeras } 2 \text { semanas y } 120 \text { en } \\
\text { última semana. }\end{array}$ & $\begin{array}{l}12 \text { semanas, } 2 \text { sesiones } \\
\text { por semana, } 20 \text { minutos } \\
\text { de duración cada sesión. }\end{array}$ & $\begin{array}{l}\text { - Fuerza muscular (dinamometría } \\
\text { prensión manual, curl-ups, salto } \\
\text { longitudinal) } \\
\text { - Agilidad (square test) } \\
\text { - Velocidad (sprint } 20 \mathrm{~m} \text { ) } \\
\text { - Flexibilidad (sit and reach) } \\
\text { - Capacidad CR (mile run test para } \\
\text { determinar Vo2 máx. estimado) } \\
\text { - Coordinación motora gruesa (KTK } \\
\text { Test) }\end{array}$ & $\begin{array}{l}\text { Grupo de intervención } \\
\text { aumentó significativamente la } \\
\text { fuerza de prensión manual, } \\
\text { flexibilidad, salto longitudinal, } \\
\text { agilidad, capacidad CR y } \\
\text { fuerza abdominal. }\end{array}$ \\
\hline $\begin{array}{l}\text { Crouter et } \\
\text { al., } 2016 .\end{array}$ & $\begin{array}{l}30 \text { niños y adolescentes } \\
\text { incluidos en el mismo } \\
\text { grupo. }\end{array}$ & $\begin{array}{l}\text { Entre } 7 \text { y } 1 \\
\text { años. }\end{array}$ & 8Obesidad & $\begin{array}{l}\text { Entrenamiento de fuerza }+ \text { ejercicio } \\
\text { aeróbico. } \\
\text { Se utilizaron maquinas, bandas elásticas y } \\
\text { peso corporal. } \\
\text { Intensidad: aproximadamente } 70 \% \text { de } 1 \text {-RM. } \\
\text { Volumen: } 8 \text { a } 12 \text { repeticiones, } 2 \text { a } 4 \text { series. }\end{array}$ & $\begin{array}{l}24 \text { semanas, } 4 \text { sesiones } \\
\text { por semana ( } 2 \text { sesiones de } \\
\text { entrenamiento de fuerza } \\
\text { en máquina Cybex), con } \\
\text { una duración de } 90 \\
\text { minutos cada sesión. }\end{array}$ & $\begin{array}{l}\text { - Capacidad CR (Frecuencia cardiaca) } \\
\text { e- Fuerza muscular (prensa de pierna y } \\
\text { prensa de pecho) } \\
\text { - Resistencia muscular (repeticiones } \\
\text { en prensa de pierna y pecho) }\end{array}$ & $\begin{array}{l}\text { La capacidad CR, fuerza y } \\
\text { resistencia muscular de pierna } \\
\text { (LP) y pecho }(\mathrm{CP}) \\
\text { aumentaron significativamente } \\
\text { en el grupo de intervención }\end{array}$ \\
\hline $\begin{array}{l}\text { Alberga et } \\
\text { al., } 2013 \text {. }\end{array}$ & $\begin{array}{l}19 \text { niños divididos en dos } \\
\text { grupos (grupo } \\
\text { experimental } n=12 / \text { grupo } \\
\text { control } n=7 \text { ) }\end{array}$ & $\begin{array}{l}\text { Entre } 8 \text { y } 1 \\
\text { años. } \\
\text { o }\end{array}$ & & $\begin{array}{l}\text { Entrenamiento de fuerza. } \\
\text { Se utilizaron mancuernas, balones } \\
\text { medicinales y máquinas para extremidad } \\
\text { superior y extremidad inferior. } \\
\text { Intensidad: } 65-85 \% \text { de } 1 \text {-RM, } 1 \text { serie, } 8-12 \\
\text { repeticiones. }\end{array}$ & $\begin{array}{l}12 \text { semanas, } 2 \text { veces por } \\
\text { semana, } 75 \text { minutos de } \\
\text { duración cada sesión. }\end{array}$ & $\begin{array}{l}\text { - Fuerza muscular (prensa de pierna y } \\
\text { prensa de brazos). }\end{array}$ & $\begin{array}{l}\text { La fuerza aumentó en el grupo } \\
\text { de intervención un } 26,8 \% \text { en } \\
\text { piernas y un } 9.8 \% \text { en brazos, } \\
\text { siendo sólo significativo el } \\
\text { cambio en piernas. }\end{array}$ \\
\hline $\begin{array}{l}\text { Calcaterra } \\
\text { et al., } 2013 .\end{array}$ & $\begin{array}{l}22 \text { niños y adolescentes } \\
\text { incluidos en el mismo } \\
\text { grupo. }\end{array}$ & $\begin{array}{l}\text { Entre } 9 \text { y } 1 \\
\text { años. }\end{array}$ & 6Obesidad & $\begin{array}{l}\text { Entrenamiento de fuerza }+ \text { ejercicio aeróbico } \\
\text { Se utilizaron balones medicinales de } 2 \text { a } 5 \mathrm{~kg} \text {. } \\
\text { Intensidad: } 60-75 \% \mathrm{FC}_{\max } \text { Circuitos de } 3-5 \\
\text { estaciones de ejercicios de fuerza y } 3-5 \\
\text { estaciones de ejercicio aeróbico de forma } \\
\text { intercalada }\end{array}$ & $\begin{array}{l}\text { o12 semanas, } 2 \text { veces por } \\
\text { semana, } 90 \text { minutos de } \\
\text { duración cada sesión. }\end{array}$ & $\begin{array}{l}\text { - Capacidad CR }\left(\mathrm{VO}_{2 \max }\right) / \text { protocolo } \\
\text { de Bruce. } \\
\text { - Fuerza muscular (dinamometría } \\
\text { prensión manual) } \\
\text { - Flexibilidad (sit and reach) }\end{array}$ & $\begin{array}{l}\text { La capacidad CR y la fuerza de } \\
\text { prensión manual aumentaron } \\
\text { significativamente. } \\
\text { La flexibilidad aumentó, pero } \\
\text { su cambio no fue significativo. }\end{array}$ \\
\hline $\begin{array}{l}\text { Vásquez et } \\
\text { al., } 2013 .\end{array}$ & $\begin{array}{l}111 \text { niños divididos en dos } \\
\text { grupos (grupo intervención } \\
\text { temprana } \mathrm{n}=60 / \text { grupo } \\
\text { intervención tardía } \mathrm{n}=51 \text { ) }\end{array}$ & & 3Obesidad & $\begin{array}{l}\text { Entrenamiento de fuerza muscular (6 grupos } \\
\text { musculares). } \\
\text { Se utilizaron mancuernas para extremidad } \\
\text { superior y peso corporal para extremidad } \\
\text { inferior. } \\
\text { Circuito de entrenamiento } 1 \times 2 \times 3: 1 \text { min de } \\
\text { trabajo x } 2 \text { min de pausa x } 3 \text { repeticiones. }\end{array}$ & $\begin{array}{l}3 \text { meses, } 3 \text { veces por } \\
\text { semana, } 45 \text { minutos de } \\
\text { duración cada sesión. }\end{array}$ & - Capacidad CR (test 6 min) & $\begin{array}{l}\text { Aumentó significativamente la } \\
\text { capacidad CR en los dos } \\
\text { grupos de intervención }\end{array}$ \\
\hline $\begin{array}{l}\text { Farris et al. } \\
2011\end{array}$ & $\begin{array}{l}, 51 \text { niños incluidos en el } \\
\text { mismo grupo. }\end{array}$ & $\begin{array}{l}\text { Entre } 8 \text { y } 1 \\
\text { años. }\end{array}$ & 2Obesidad & $\begin{array}{l}\text { Entrenamiento de fuerza }+ \text { actividad } \\
\text { aeróbica. } \\
\text { Se utilizaron máquinas de resistencia } \\
\text { neumática, peso corporal para el } \\
\text { entrenamiento y ejercicios de estiramientos. } \\
\text { Intensidad: 6-7 en una escala de esfuerzo } \\
\text { percibido de } 0 \text { a } 10 \text {. }\end{array}$ & $\begin{array}{l}\text { d12 semanas, } 2 \text { sesione } \\
\text { por semana (obligatoria) } \\
\text { iaduración } 60 \text { minutos cade } \\
\text { elsesión. Más } 1 \text { sesiór } \\
\text { lúdica. } \\
\text { o }\end{array}$ & $\begin{array}{l}\text { s- Flexibilidad (sit and reach). } \\
\text {,- Fuerza muscular (push-ups / curl- } \\
\text { aups) } \\
\text { n- CR (step test) }\end{array}$ & $\begin{array}{l}\text { La flexibilidad, función } \\
\text { muscular y función CR } \\
\text { aumentaron } \\
\text { significativamente. }\end{array}$ \\
\hline
\end{tabular}

En cuatro de los estudios, los participantes incluidos fueron exclusivamente niños (menores de 12 años) (Alberga et al., 2013; Farris et al., 2011; Nobre et al., 2017; Vasquez et al., 2013), sin embargo, en el resto de los artículos hubo una combinación de niños y adolescentes en la muestra (Bruno et al., 2018; Calcaterra et al., 2013; Crouter et al., 2017; Horsak et al., 2019). En todos los artículos seleccionados se incluyeron niños con estado nutricional de obesidad. En los estudios de Bruño et al. (2018) y Nobre et al. (2017), además, se incluyeron niños con sobrepeso.

\section{Protocolos de entrenamiento y duración}

Tres estudios intervinieron exclusivamente con un entrenamiento de fuerza (Alberga et al., 2013; Nobre et al., 2017; Vasquez et al., 2013), no obstante, fueron métodos diferentes. Nobre et al. (2017) aplicaron un entrenamiento de tipo pliométrico basado en saltos sobre cajones, mientras que Alberga et al. (2013) entrenaron a los niños contra resistencia utilizando mancuernas y maquinas. Por su parte, Vásquez et al. (2013) realizaron un circuito denominado 1 x 2 x 3 donde los niños tenían que realizar estaciones de ejercicios durante 1 minuto de trabajo de fuerza (con mancuernas y peso corporal), 2 minutos de pausa y 3 series. En el estudio de Horsak et al. (2018), el entrenamiento de fuerza fue combinado con ejercicios neuromusculares, utilizando el peso corporal. Los cuatro estudios restantes combinaron entrenamientos de fuerza con actividades aeróbicas (Bruno et al., 2018; Calcaterra et al., 2013; 
Crouter et al., 2017; Farris et al., 2011). De estos, tres estudios utilizaron mancuernas, maquinas y peso corporal para los protocolos de fuerza (Bruno et al., 2018; Crouter et al., 2017; Farris et al., 2011) y uno utilizó balones medicinales de 2 a $5 \mathrm{~kg}$ (Calcaterra et al., 2013).

Coincidentemente, siete de los estudios seleccionados aplicaron un programa de entrenamiento de 12 semanas de duración con 2 a 5 sesiones semanales (Alberga et al., 2013; Bruno et al., 2018; Calcaterra et al., 2013; Farris et al., 2011; Horsak et al., 2019; Nobre et al., 2017; Vasquez et al., 2013). Por su parte, el estudio de Crouter et al. (2016) intervino durante 24 semanas con 4 sesiones semanales. Todos los estudios seleccionados utilizaron un tiempo de 45 a 90 minutos por sesión, excepto el estudio de Nobre et al. (2017), que dedicó 20 minutos a la intervención (entrenamiento pliométrico).

Con respecto a la intensidad utilizada por las investigaciones, es bastante variable su monitorización. Dos de los estudios utilizaron intensidades basadas en una repetición máxima (1RM), uno de ellos utilizó 70\% de 1 RM (Crouter et al., 2017) y el otro entre el 65-85\% de 1 RM (Alberga et al., 2013). Un estudio utilizó la escala de esfuerzo percibido (Farris et al., 2011) y uno utilizó la $\mathrm{FC}_{\text {max }}$ (Calcaterra et al., 2013). El resto de los estudios no especifica la intensidad utilizada en los entrenamientos (Bruno et al., 2018; Horsak et al., 2019; Nobre et al., 2017; Vasquez et al., 2013). En el caso del estudio llevado a cabo por Nobre et al. (2017), si bien no se señala la intensidad del entrenamiento, se detalla específicamente el volumen utilizado semana a semana (5 series de 10-24 repeticiones progresivas a lo largo de las semanas con un total de 50 saltos, 50 en las primeras 2 semanas y 120 saltos en última semana).

\section{Variables de condición física}

La fuerza muscular fue evaluada por 7 estudios (Alberga et al., 2013; Bruno et al., 2018; Calcaterra et al., 2013; Crouter et al., 2017; Farris et al., 2011; Horsak et al., 2019; Nobre et al., 2017), mientras que la capacidad cardiorrespiratoria fue analizada por 6 de los estudios (Bruno et al., 2018; Calcaterra et al., 2013; Crouter et al., 2017; Farris et al., 2011; Nobre et al., 2017; Vasquez et al., 2013). Por su parte, la flexibilidad fue considerada por 3 estudios (Calcaterra et al., 2013; Farris et al., 2011; Nobre et al., 2017). Mientras que, otras variables de la condición física fueron la agilidad (Nobre et al., 2017), velocidad (Nobre et al., 2017), coordinación motora gruesa (Nobre et al., 2017) y resistencia a la fatiga muscular (Crouter et al., 2017).
Para obtener la fuerza muscular del tren superior, los estudios utilizaron la prueba de prensión manual (Bruno et al., 2018; Calcaterra et al., 2013; Nobre et al., 2017), prensa de brazos (Alberga et al., 2013; Crouter et al., 2017) y flexiones de brazos (Farris et al., 2011). Para medir la fuerza muscular del tren inferior se utilizó la prueba de prensa de piernas (Alberga et al., 2013; Crouter et al., 2017), salto longitudinal y mediciones especificas a grupos musculares como cuádriceps (Horsak et al., 2019), abductores de cadera (Horsak et al., 2019) y abdominales (curl-ups) (Farris et al., 2011; Nobre et al., 2017). En relación con la evaluación de la capacidad cardiorrespiratoria, se utilizaron pruebas directas de consumo máximo de oxígeno (Bruno et al., 2018; Calcaterra et al., 2013), la prueba de 6 minutos de marcha (Vasquez et al., 2013), la prueba de correr una milla (Nobre et al., 2017) y el step test (Farris et al., 2011). Para medir la flexibilidad del tren inferior todos los estudios que la consideraron emplearon la prueba de sit and reach (Calcaterra et al., 2013; Farris et al., 2011; Nobre et al., 2017).

\section{Principales resultados obtenidos por los estu-} dios

Los estudios señalan que el entrenamiento de fuerza cuando es aplicado de manera aislada aumenta la fuerza muscular (Alberga et al., 2013; Nobre et al., 2017), flexibilidad (Nobre et al., 2017), potencia muscular (Nobre et al., 2017), agilidad (Nobre et al., 2017) y capacidad cardiorrespiratoria (Nobre et al., 2017; Vasquez et al., 2013) en niños y niñas. La intervención que incorporó un entrenamiento de tipo pliométrico aumentó significativamente la fuerza de prensión manual, flexibilidad, salto longitudinal, agilidad, capacidad cardiorrespiratoria y fuerza abdominal (Nobre et al., 2017), mientras que los estudios con entrenamiento de fuerza tradicional mostraron aumentos significativos de la fuerza de piernas (Alberga et al., 2013) y la capacidad cardiorrespiratoria (Vasquez et al., 2013).

Cuando el entrenamiento de fuerza se combinó con otras intervenciones también se reportaron cambios positivos en las variables de la condición física. De esta manera, la fuerza combinada con el entrenamiento neuromuscular evidenció un aumento de la fuerza en cuádriceps y abductores, consiguiendo un incremento significativo solo para este último grupo muscular (Horsak et al., 2019). Por otra parte, cuando el entrenamiento de fuerza se combinó con actividades aeróbicas se observaron aumentos significativos de la fuerza (Bruno et al., 2018; Calcaterra et al., 2013; Crouter et al., 2017; 
Farris et al., 2011), capacidad cardiorrespiratoria (Bruno et al., 2018; Calcaterra et al., 2013; Crouter et al., 2017; Farris et al., 2011) y flexibilidad (Calcaterra et al., 2013; Farris et al., 2011).

\section{Discusión}

El objetivo de esta revisión sistemática fue analizar la evidencia científica del entrenamiento de fuerza muscular sobre la condición física en niños con sobrepeso y obesidad. Como principales hallazgos se encuentran que el entrenamiento de fuerza, de manera aislada o combinada, provoca un efecto positivo y significativo sobre algunas variables de la condición física, especialmente, a nivel de fuerza muscular, capacidad cardiorrespiratoria, flexibilidad, potencia muscular y agilidad en niños y niñas con sobrepeso y obesidad.

\section{Intensidad y volumen del entrenamiento de} fuerza

Para controlar la intensidad del entrenamiento de fuerza, los estudios revisados reportan la utilización de una escala de esfuerzo percibido OMNI-RES $(0-10)$ (Farris et al., 2011; Horsak et al., 2019). Específicamente, en la investigación de Farris, et al. (2011) se establecieron valores de 6-7 como intensidad de la intervención, que equivale, aproximadamente, al $75 \%$ de 1RM según la conversión sugerida en la literatura (Helms, Cronin, Storey, \& Zourdos, 2016; Zourdos et al., 2016). Por su parte, Crouter, et al. (2016) trabajaron con el 70\% de 1 RM en máquinas guiadas, bandas elásticas y peso corporal durante el entrenamiento de fuerza. Mientras que Alberga, et al. (2013) determinaron una intensidad entre el 65-85\% de 1 RM en los ejercicios sobre maquinas guiadas para su intervención. Similares intensidades son utilizadas en adolescentes y adultos obesos, donde se describen las primeras sesiones en un 65-70\% de 1RM y una progresión hasta el 8090\% de 1RM (Bouchard, Soucy, Senechal, Dionne, \& Brochu, 2009; Clark \& Goon, 2015; Kraemer et al., 1999; Shaibi et al., 2006).

Para establecer el volumen del entrenamiento, los estudios describen esta variable a través de la utilización de series y repeticiones. Nobre, et al. (2017) trabajaron con un volumen de 5 series con 10 a 24 repeticiones progresivas durante el transcurso del entrenamiento, mientras que Crouter, et al. (2016) entrenaron con 2 a 4 series de 8 a 12 repeticiones por ejercicio. Por su parte, Alberga, et al. (2013) utilizaron 1 serie de 8 a 12 repeticiones como volumen de entrenamiento durante toda la intervención. En adolescentes y adultos obesos, los estudios han reportado la utilización de 18 30 repeticiones divididas en 3 a 6 series según las características iniciales de los participantes y progresión de la intervención (Bouchard et al., 2009; Clark \& Goon, 2015; Kraemer et al., 1999; Shaibi et al., 2006).

\section{Fuerza Muscular}

Independientemente, si el método de entrenamiento de fuerza utilizado fue tradicional (Alberga et al., 2013; Vasquez et al., 2013), pliométrico (Nobre et al., 2017), combinado con ejercicio aeróbico (Bruno et al., 2018; Calcaterra et al., 2013; Crouter et al., 2017; Farris et al., 2011) o combinado con ejercicio neuromuscular (Horsak et al., 2019), los estudios revisados reportaron un aumento de la fuerza muscular en niños y niñas con sobrepeso y obesidad. El tren inferior reportó un aumento de la fuerza en las pruebas de prensa de pierna (Alberga et al., 2013; Crouter et al., 2017) y salto longitudinal (Nobre et al., 2017). Además, en otra de las investigaciones se presentó un aumento de la fuerza de cuádriceps en 20\% y fuerza de abductores de cadera en 30\%, sin embargo, el cambio fue significativo solo en el último grupo muscular (Horsak et al., 2019). Por su parte, el tren superior, exhibió un aumento de la fuerza en las evaluaciones de prensión manual (Bruno et al., 2018; Calcaterra et al., 2013; Nobre et al., 2017), prensa de brazos (Crouter et al., 2017) y flexiones de bazos (Farris et al., 2011). Además, la resistencia abdominal aumentó en la prueba de abdominales (curl-ups) (Farris et al., 2011; Nobre et al., 2017). En adolescentes y adultos obesos, el entrenamiento de fuerza también ha mostrado efectos positivos en la fuerza muscular (Borde, Hortobagyi, \& Granacher, 2015; Dias et al., 2015; Kim et al., 2016), incluso cuando se utilizan métodos de entrenamiento de fuerza pliométricos (Fischetti, Cataldi, \& Greco, 2019) o combinados con ejercicio aeróbico (Jin, Rhyu, \& Kim, 2018).

\section{Capacidad Cardiorrespiratoria}

Los estudios revisados revelaron un aumento de la capacidad cardiorrespiratoria cuando se aplicó un protocolo de fuerza de forma aislada (Nobre et al., 2017; Vasquez et al., 2013) o combinada (Bruno et al., 2018; Calcaterra et al., 2013; Crouter et al., 2017; Farris et al., 2011) con entrenamiento aeróbico en niños y niñas con sobrepeso y obesidad. Similares resultados se han reportado en adolescentes y adultos con sobrepeso y obesidad, informando que el entrenamiento de fuerza aislado (Chen, Ismail, \& Al-Safi, 2016) o combinado 
(Alberga et al., 2016; Kim, 2009) mejoran la capacidad cardiorrespiratoria.

Otro elemento relevante de los estudios analizados en nuestra revisión y que incluyeron entrenamiento de fuerza aislada, se refiere al uso de distintos métodos de intervención. Vásquez et al. (2013) por medio de circuitos aplicaron un entrenamiento de fuerza a niños de 8-12 años enfocado en 6 grupos musculares: bíceps, hombros, pectorales, abdominales, gemelos y muslos. Este protocolo se realizó durante 3 meses con una frecuencia de 3 sesiones semanales en días no consecutivos, con una duración de 45 minutos por sesión. El aumento de la capacidad cardiorrespiratoria fue evaluada a través de la distancia recorrida en la prueba de 6 minutos (Vasquez et al., 2013). La investigación llevada a cabo por Nobre et al. (2017) aplicó un entrenamiento de fuerza pliométrico durante 12 semanas, con una frecuencia de 2 sesiones semanales de 20 minutos por sesión, en la cual se determinó el aumento de la capacidad cardiorrespiratoria con la prueba de carrera de 1 milla (Nobre et al., 2017). Se ha informado que la aplicación de estímulos anaeróbicos a partir de ejercicios de fuerza de alta intensidad y corta duración, permite movilizar depósitos de glucógeno, con los consecuentes cambios intracelulares que promueven la síntesis de proteínas estructurales y funcionales responsables de mejorar la capacidad funcional del músculo, lo cual, podría contribuir en la eficiencia del consumo máximo de oxígeno (Vasquez et al., 2013).

\section{Flexibilidad}

Se ha demostrado que el entrenamiento de fuerza favorece una mayor flexibilidad de la región lumbopélvica-isquiosurales en niños con sobrepeso y obesidad (Calcaterra et al., 2013; Farris et al., 2011; Nobre et al., 2017). Esto evaluado a través de la prueba sit and reach y bajo protocolos de fuerza pliométricos (Nobre et al., 2017) y combinados (Calcaterra et al., 2013; Farris et al., 2011). La mejora de la flexibilidad luego de una intervención se ha asociado, principalmente, al entrenamiento de fuerza. La literatura señala que los ejercicios de fuerza aumentan la masa y la contractilidad muscular, además de mejorar la fuerza tensil de tendones y ligamentos, lo cual permitiría alcanzar un mayor rango de movimiento en las articulaciones durante una prueba de flexibilidad (Kim, 2009). Además, en modelos animales se ha visto que el entrenamiento de fuerza aumenta el número de fibroblastos activos en el tendón y mejora la síntesis de colágeno, favoreciendo la elasticidad de estos tejidos (Said et al., 2018).

\section{Otras variables relacionadas con la condición} física

En esta revisión también se encontró que el entrenamiento de fuerza mostró resultados positivos sobre la agilidad (Nobre et al., 2017), balance (Nobre et al., 2017), coordinación (Nobre et al., 2017) y resistencia a la fatiga muscular (Crouter et al., 2017) en niños y niñas con sobrepeso y obesidad, principalmente, con la implementación de actividades pliométricas. En adultos jóvenes con obesidad, se han observado resultados similares luego de participar en intervenciones con entrenamiento de fuerza tradicional, informando mejoras significativas en la velocidad y agilidad (Said et al., 2018). Es probable que tanto en niños como adultos, un entrenamiento de fuerza sea capaz de generar estímulos suficientes en el músculo para provocar adaptaciones neurales que favorezcan la fisiología de las unidades motoras (Guzmán-Muñoz et al., 2020b; Guzmán-Muñoz et al., 2020c), lo que podría explicar una mejora en el rendimiento de variables como el balance, agilidad y coordinación.

\section{Conclusiones}

En esta revisión sistemática se puede concluir que el entrenamiento de fuerza es un método eficaz para mejorar la condición física de niños y niñas con sobrepeso y obesidad. Pese a la escasa evidencia y a la diversidad de métodos de entrenamiento encontrados en nuestra revisión, recomendamos el uso del entrenamiento de fuerza combinado con otras estrategias de actividad física de intensidad moderada a vigorosa, respetando la experiencia, edad, intereses y patologías de los niños y niñas con sobrepeso y obesidad. La aplicación práctica de esta revisión es proveer a los profesionales de las ciencias de la salud y del deporte las mejores opciones de entrenamiento basadas en la evidencia científica para la población infantil.

\section{Referencias}

Alberga, A. S., Farnesi, B. C., Lafleche, A., Legault, L., \& Komorowski, J. (2013). The Effects of Resistance Exercise Training on Body Composition and Strength in Obese Prepubertal Children. Physician and Sportsmedicine, 41(3), 103-109. doi: https: / / doi.org/ 10.3810/psm.2013.09.2028

Alberga, A. S., Prud'homme, D., Sigal, R. J., Goldfield, G. S., Hadjiyannakis, S., Phillips, P., ... Gougeon, R. (2016). Effects of aerobic training, resistance 
training, or both on cardiorespiratory and musculoskeletal fitness in adolescents with obesity: the HEARTY trial. Applied Physiology, Nutrition, and Metabolism, 41(3), 255-265. doi: https://doi.org/ 10.1139/apnm-2015-0413

Bastien, M., Poirier, P., Lemieux, I., \& Despres, J. P. (2014). Overview of Epidemiology and Contribution of Obesity to Cardiovascular Disease. Progress in Cardiovascular Diseases, 56(4), 369-381. doi: https://doi.org/10.1016/j.pcad.2013.10.016

Borde, R., Hortobagyi, T., \& Granacher, U. (2015). Dose-Response Relationships of Resistance Training in Healthy Old Adults: A Systematic Review and Meta-Analysis. Sports Medicine, 45(12), 1693-1720. doi: https://doi.org/10.1007/s40279-015-0385-9

Bouchard, D. R., Soucy, L., Senechal, M., Dionne, I. J., \& Brochu, M. (2009). Impact of resistance training with or without caloric restriction on physical capacity in obese older women. Menopause-the Journal of the North American Menopause Society, 16(1), 66-72. doi: https://doi.org/10.1097/ gme.0b013e31817dacf7

Bruno, A., Escobar, P., Cebolla, A., Alvarez-Pitti, J., Guixeres, J., Lurbe, E., . . Lison, J. F. (2018). Homeexercise Childhood Obesity Intervention: A Randomized Clinical Trial Comparing Print Versus Web-based (Move It) Platforms. Journal of Pediatric Nursing-Nursing Care of Children \& Families, 42, E79E84. doi: https://doi.org/10.1016/ j.pedn.2018.04.008

Calcaterra, V., Larizza, D., Codrons, E., De Silvestri, A., Brambilla, P., Abela, S., .. . Vandoni, M. (2013). Improved metabolic and cardiorespiratory fitness during a recreational training program in obese children. Journal of Pediatric Endocrinology \& Metabolism, 26(3-4), 271-276. doi: https://doi.org/10.1515/ jpem-2012-0157

Chen, C. K., Ismail, N. S., \& Al-Safi, A. A. (2016). Effects of brisk walking and resistance training on cardiorespiratory fitness, body composition, and lipid profiles among overweight and obese individuals. Journal of Physical Education and Sport, 16(3), 957-963.

Clark, J., \& Goon, D. (2015). The role of resistance training for treatment of obesity related health issues and for changing health status of the individual who is overfat or obese: a review. The Journal of sports medicine and physical fitness, 55(3), 205-222.

Crouter, S. E., Salas, C., \& Wiecha, J. (2017). Effects of an afterschool community center physical activity program on fitness and body composition in obese youth. Journal of Sports Sciences, 35(11), doi: https:/ / doi.org/1034-1040.

doi:10.1080/

02640414.2016.1209305

de Onis, M., Blossner, M., \& Borghi, E. (2010). Global prevalence and trends of overweight and obesity among preschool children. American Journal of Clinical Nutrition, 92(5), 1257-1264. doi: doi: https:// doi.org/10.3945/ajcn.2010.29786

Dias, I., Farinatti, P., Gracas Coelho De Souza, M. D., Manhanini, D. P., Balthazar, E., Simplicio Dantas, D. L., . . Kraemer-Aguiar, L. G. (2015). Effects of Resistance Training on Obese Adolescents. Medicine and Science in Sports and Exercise, 47(12), 2636-2644. doi: https://doi.org/10.1249/ mss.0000000000000705

Faigenbaum, A., Perez, T., \& Naclerio, F. (2011). Resistance training for overweight youth. Revista Kronos, 10(1), 5-14.

Farris, J.W., Taylor, L., Williamson, M., \& Robinson, C. (2011). A 12-week interdisciplinary intervention program for children who are obese. Cardiopulmonary physical therapy journal, 22(4), 12-20.

Fischetti, F., Cataldi, S., \& Greco, G. (2019).A combined plyometric and resistance training program improves fitness performance in 12 to 14 -years-old boys. Sport Sciences for Health, 15(3), 615-621. doi: https://doi.org/10.1007/s11332-019-00560-2

Fraser, B. J., Schmidt, M. D., Huynh, Q. L., Dwyer, T., Venn, A. J., \& Magnussen, C. G. (2017). Tracking of muscular strength and power from youth to young adulthood: Longitudinal findings from the Childhood Determinants of Adult Health Study. Journal of Science and Medicine in Sport, 20(10), 927-931. doi: https:// doi.org/10.1016/j.jsams.2017.03.021

Grontved, A., Ried-Larsen, M., Moller, N. C., Kristensen, P. L., Froberg, K., Brage, S., \& Andersen, L. B. (2015). Muscle strength in youth and cardiovascular risk in young adulthood (the European Youth Heart Study). British Journal of Sports Medicine, 49(2), 90-U38. doi: https://doi.org/10.1136/ bjsports-2012-091907

Guzmán-Muñoz, E., \& Castillo-Retamal, M. (2020a). Control postural en niños con sobrepeso y obesidad: una revisión de la literatura. Revista Cientifica Salud Uninorte, 36(2),471-488. doi: http://dx.doi.org/ 10.14482/sun.36.2.616.398

Guzmán-Muñoz, E., Sazo-Rodriguez, S., Concha-Cisternas, Y., Valdes-Badilla, P., Lira-Cea, C., SilvaMoya, G., . . . Mendez-Rebolledo, G. (2020b). Four Weeks of Neuromuscular Training Improve Static 
and Dynamic Postural Control in Overweight and Obese Children: A Randomized Controlled Trial. Journal of Motor Behavior,52(6),761-769. doi: https: / /doi.org/10.1080/00222895.2019.1694486

Guzmán-Muñoz, E., Valdés-Badilla, P., Concha-Cisternas, Y., Méndez-Rebolledo, G., \& Castillo Retamal, M. (2020c). Methods for measuring physical activity in children and their relationship with nutritional status: a narrative review. Archivos de medicina del deporte,37(3), 197-203.

Guzmán-Muñoz, E., Valdes-Badilla, P., MendezRebolledo, G., Concha-Cisternas, Y., \& CastilloRetamal, M. (2019). Relationship between anthropometry and balance of postural control in children 6-9 years old. Nutricion Hospitalaria, 36(1), 32-38. doi: https: / / doi.org/10.20960/nh.02072

He, H., Pan, L., Dui, J., Liu, F., Jin, Y., Ma, J., . . . Shan, G. (2019). Muscle fitness and its association with body mass index in children and adolescents aged 7-18 years in China: a cross-sectional study. Bmc Pediatrics, 19, 101 (2019). doi: https://doi.org/10.1186/ s12887-019-1477-8

Helms, E. R., Cronin, J., Storey, A., \& Zourdos, M. C. (2016). Application of the Repetitions in ReserveBased Rating of Perceived Exertion Scale for Resistance Training. Strength and Conditioning Journal, 38(4), 42-49. doi: https://doi.org/10.1519/ ssc.0000000000000218

Horsak, B., Schwab, C., Baca, A., Greber-Platzer, S., Kreissl, A., Nehrer, S., . . . Wondrasch, B. (2019). Effects of a lower extremity exercise program on gait biomechanics and clinical outcomes in children and adolescents with obesity: A randomized controlled trial. Gait \& posture, 70, 122-129. https: / /doi.org/10.1016/j.gaitpost.2019.02.032

Jin, C.-H., Rhyu, H.-S., \& Kim, J.Y. (2018). The effects of combined aerobic and resistance training on inflammatory markers in obese men. Journal of Exercise Rehabilitation, 14(4), 660-665. doi: https: / / doi.org/10.12965/jer. 1836294.147

Kakebeeke, T. H., Lanzi, S., Zysset, A. E., Arhab, A., Messerli-Bürgy, N., Stuelb, K., . . Puder, J. J. (2017). Association between Body Composition and Motor Performance in Preschool Children. Obesity Facts, 10(5), 420-431. doi: https://doi.org/10.1159/ 000477406

Kim, H.-J., Lee, H.-J., So, B., Son, J. S., Yoon, D., \& Song, W. (2016). Effect of Aerobic Training and Resistance Training on Circulating Irisin Level and Their Association With Change of Body Composition in Overweight/Obese Adults: a Pilot Study. Physiological Research, 65(2), 271-279. doi: https: / / doi.org/10.33549/physiolres.932997

Kim, S.-Y. (2009). Effects of Combined Aerobic and Resistance Training on Cardiorespiratory Fitness in Obese Middle-aged Adults. Journal of Sport and Leisure Studies, 37(2), 1069-1080.

Kraemer, W. J., Volek, J. S., Clark, K. L., Gordon, S. E., Puhl, S. M., Koziris, L. P., . . Sebastianelli, W. J. (1999). Influence of exercise training on physiological and performance changes with weight loss in men. Medicine and Science in Sports and Exercise, 31(9), 1320 1329. doi: https://doi.org/10.1097/00005768199909000-00014

Martins, J., Cardoso, J., Honório, S., \& Silva, A. (2019). The Effect of a Strength Training Programme in Adolescents in Physical Education Classes (El efecto de un programa de entrenamiento de fuerza en adolescentes en clases de educación física). Retos, 38, 71 76. doi: https://doi.org/10.47197/ retos.v38i38.72221

Moher, D., Shamseer, L., Clarke, M., Ghersi, D., Liberati, A., Petticrew, M., . . . Grp, P. (2015). Preferred reporting items for systematic review and meta-analysis protocols (PRISMA-P) 2015 statement. Systematic Reviews, 4(1),1-9. doi: https: / / doi.org/10.1186/2046-4053-4-1

Moseley, A. M., Herbert, R. D., Sherrington, C., \& Maher, C. G. (2002). Evidence for physiotherapy practice: A survey of the Physiotherapy Evidence Database (PEDro). Australian Journal of Physiotherapy, 48(1), 43-49. doi: https: / / doi.org/10.1016/s00049514(14)60281-6

Ng, M., Fleming,T., Robinson, M., Thomson, B., Graetz, N., Margono, C., . . G Gakidou, E. (2014). Global, regional, and national prevalence of overweight and obesity in children and adults during 1980-2013: a systematic analysis for the Global Burden of Disease Study 2013. Lancet, 384(9945), 766-781. doi: https: / /doi.org/10.1016/s0140-6736(14)60460-8

Nobre, G. G., de Almeida, M. B., Nobre, I. G., dos Santos, F. K., Brinco, R. A., Arruda-Lima, T. R., . . . Moura-dos-Santos, M. A. (2017). Twelve weeks of plyometric training improves motor performance of 7-to 9-year-old boys who were overweight/obese: a randomized controlled intervention. Journal of Strength and Conditioning Research, 31(8), 2091-2099. doi: https://doi.org/10.1519/ jsc.0000000000001684

Prieto González, P., Sagat, P., Ben Brahim, M., \& 
Sedlacek, J. (2019). Análisis de la veracidad de determinadas creencias asociadas habitualmente al entrenamiento de fuerza. Una revisión narrativa (Analysis of the veracity of certain beliefs frequently associated to resistance training. A narrative review). Retos, 38, 773-781. doi: https://doi.org/10.47197/ retos.v38i38.69739

Rajmil, L., Bel, J., Clofent, R., Cabezas, C., Castell, C., \& Espallargues, M. (2016). [Clinical interventions in overweight and obesity: a systematic literature review 2009-2014]. Anales de pediatría. 86(4),197-212. doi: https: / /doi.org/10.1016/j.anpedi.2016.03.012

Ramirez-Izcoa, A., Enrique Sanchez-Sierra, L., MejiaIrias, C., Izaguirre Gonzalez, A. I., Alvarado-Avilez, C., Flores-Moreno, R., . . . Rivera, E. E. (2017). Prevalence of pediatric overweight and obesity and associated factors in public and private schools in Tegucigalpa, Honduras. Revista Chilena De Nutrición, 44(2), 161-169. doi: https://doi.org/10.4067/ s0717-75182017000200007

Ribeiro, C. C., Gómez-Conesa, A., \& Montesinos, M. H. (2010). Metodología para la adaptación de instrumentos de evaluación. Fisioterapia, 32(6), 264-270. doi: https://doi.org/10.1016/j.ft.2010.05.001

Said, M. A., Abdelmoneem, M., Almaqhawi, A., Kotob, A. A. H., Alibrahim, M. C., \& Bougmiza, I. (2018). Multidisciplinary approach to obesity: Aerobic or resistance physical exercise?. Journal of Exercise Science \& Fitness, 16(3), 118-123. doi: https://doi.org/ 10.1016/j.jesf.2018.11.001

Sanchez-Cruz, J. J., Jimenez-Moleon, J. J., FernandezQuesada, F., \& Sanchez, M. J. (2013). Prevalence of Child and Youth Obesity in Spain in 2012. Revista Española De Cardiologia, 66(5), 371-376. doi: https:/ /doi.org/10.1016/j.rec.2012.10.012

Shaibi, G. Q., Cruz, M. L., Ball, G. D. C., Weigensberg, M. J., Salem, G. J., Crespo, N. C., \& Goran, M. I. (2006). Effects of resistance training on insulin sensitivity in overweight Latino adolescent males. Medicine and Science in Sports and Exercise, 38(7), 12081215. doi: https://doi.org/10.1249/ 01.mss.0000227304.88406.0f

Sun, F., Wang, L.-J., \&Wang, L. (2015). Effects of weight management program on postural stability and neuromuscular function among obese children: study protocol for a randomized controlled trial. Trials, 16,143. doi: https://doi.org/10.1186/s13063-0150673-6

Takken, T., Elst, E., Spermon, N., Helders, P. J. M., Prakken, A. B. J., \& van der Net, J. (2003). The physiological and physical determinants of functional ability measures in children with juvenile dermatomyositis. Rheumatology, 42(4), 591-595. doi: https://doi.org/10.1093/rheumatology/keg210

Timpka, S., Petersson, I. F., Zhou, C., \& Englund, M. (2014). Muscle strength in adolescent men and risk of cardiovascular disease events and mortality in middle age: a prospective cohort study. Bmc Medicine, 12,62. doi: https://doi.org/10.1186/1741-7015$12-62$

Tobarra, S. E., Castro, O. Ó., \& Badilla, C. R. (2015). Estado nutricional y características socioepidemiológicas de escolares chilenos, OMS 2007. Revista chilena de pediatría, 86(1), 12-17. doi: http://dx.doi.org/10.1016/j.rchipe.2015.04.003 Vasquez, F., Diaz, E., Lera, L., Meza, J., Salas, I., Rojas, P., . . Burrows, R. (2013). Residual effect of muscle strength exercise in secondary prevention of childhood obesity. Nutrición Hospitalaria, 28(2), 333339. doi: http://dx.doi.org/10.3305/ nh.2013.28.2.6159

Wind, A. E., Takken, T., Helders, P. J. M., \& Engelbert, R. H. H. (2010). Is grip strength a predictor for total muscle strength in healthy children, adolescents, and young adults?. European Journal of Pediatrics, 169(3), 281-287. doi: http://dx.doi.org/ 10.1007/s00431-009-1010-4

Zourdos, M. C., Klemp, A., Dolan, C., Quiles, J. M., Schau, K. A., Jo, E., . . . Blanco, R. (2016). novel resistance training-specific rating of perceived exertion scale measuring repetitions in reserve. Journal of Strength and Conditioning Research, 30(1), 267-275. doi: http://dx.doi.org/10.1519/ jsc.0000000000001049

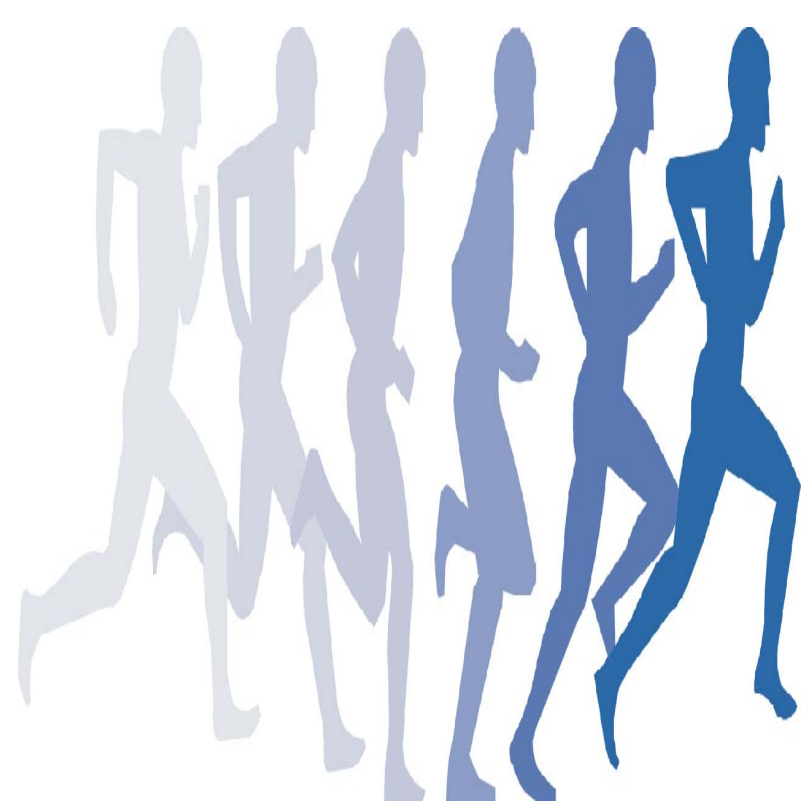

